\title{
Primeiro registro de Eufriesea laniventris (Ducke, 1902) (Hymenoptera, Apidae, Euglossini) no Amazonas, Brasil*.
}

\author{
Eliana Fernandez STORTI ${ }^{1}{ }^{* *}$, Atilio STORTI FILHO ${ }^{2}$, Márcio Luiz de OLIVEIRA ${ }^{3}$.
}

\begin{abstract}
RESUMO
É registrada pela primeira vez a ocorrência de Eufriesea laniventris no Amazonas. No ano 2000, foram coletados seis exemplares na região de Manaus ( $2^{\circ} 36^{\prime} \mathrm{S}$ e $60^{\circ} 02^{\prime} \mathrm{W}$ ) atraídos pelas substâncias odoríferas 1,8 cineol e salicilato de metila.
\end{abstract}

PALAVRAS-CHAVE:

abelhas, distribuição geográfica, novos registros.

\section{First record of Eufriesea laniventris (Ducke, 1902) (Hymenoptera, Apidae, Euglossini) in the state of Amazonas, Brazil.}

\begin{abstract}
The first record of Eufriesea laniventris in the state of Amazonas is bere reported. Attracted to 1.8 cineole and methyl salicylate fragrances, six specimens were collected in the region of Manaus (2० $\left.36560^{\circ} 02^{\prime} \mathrm{W}\right)$ during the year 2000.
\end{abstract}

KEY WORDS

geographic distribution, new records, orchid bees.

O primeiro estudo utilizando iscas odoríferas para a captura de abelhas Euglossini, no Amazonas, foi o de Braga (1976) em região de campina, campinarana e floresta tropical úmida próximas à Manaus. A maioria dos estudos posteriores aconteceu nas áreas do Projeto Dinâmica Biológica de Fragmentos Florestais (Convênio INPA/Smithsonian Institution). Powel \& Powell (1987) verificaram que para a maioria das espécies, a taxa de visitas a iscas de substâncias odoríferas diminuiu com o tamanho do fragmento. De modo contrário, Becker et al. (1991) verificaram que a abundância das abelhas foi maior nos fragmentos de $10 \mathrm{e}$ 100 ha do que em mata contínua. Morato (1994), por sua vez, comparou a fauna de Euglossini da mata contínua, borda e capoeira e constatou que embora a abundância tenha decrescido da mata para a capoeira e a riqueza tenha diferido pouco entre esses ambientes, o baixo valor da similaridade encontrado entre a mata e a capoeira sugere que o desmatamento esteja afetando a fauna de Euglossini. Por fim, Oliveira \& Campos (1995) e Oliveira (2001) constataram que a diversidade de Euglossini difere até mesmo de uma área de mata contínua para outra, em uma mesma floresta.
O resultado aqui apresentado é parte de dois projetos (PPI 1-3030 e PPI 1-3590) que têm por objetivo conhecer a fauna de invertebrados que habita fragmentos florestais na região de Manaus. Duas das áreas estudadas são de floresta primária: Estação Experimental de Silvicultura Tropical - 180.000ha e a Reserva Florestal Adolpho Ducke - 10.072ha e quatro são fragmentos florestais na área urbana: Universidade Federal do Amazonas - 536ha, Fazenda IPE - 100ha, Parque Municipal do Mindu - 33ha e Instituto Nacional de Pesquisas da Amazônia/CPAQ 13ha. Em cada uma delas foram realizadas cinco coletas durante o ano 2000.

As substâncias utilizadas para a captura das abelhas foram acetato de benzila, 1,8 cineol, eugenol, salicilato de metila e vanilina, colocadas em pavios de gaze inseridos em tubos de ensaio de plástico para evitar sua rápida evaporação. Os tubos foram pendurados a uma distância de aproximadamente $2,0 \mathrm{~m}$ entre si e a $1,5 \mathrm{~m}$ do solo, sobre arbustos ou árvores presentes na área, no horário das 08:00 às 12:00 horas.

\footnotetext{
* Trabalho parcialmente financiado pelo PPI 1-3030 e PPI 1-3590.

** Corresponding author e-mail: storti@inpa.gov.br

${ }^{1}$ Instituto Nacional de Pesquisas da Amazônia - INPA, Coordenação de Pesquisas em Ecologia, Caixa Postal 478, 69061-970, Manaus, AM.

${ }^{2}$ Instituto Nacional de Pesquisas da Amazônia - INPA, Coordenação de Pesquisas em Aqüicultura.

${ }^{3}$ Instituto Nacional de Pesquisas da Amazônia - INPA, Coordenação de Pesquisas em Entomologia.
} 


\section{ACTA AMAZONICA}

PRIMEIRO REGISTRO DE EUFRIESEA LANIVENTRIS (DUCKE, 1902) (HYMENOPTERA, APIDAE, EUGLOSSINI) NO AMAZONAS, BRASIL
Ao se aproximarem da isca, as abelhas foram coletadas individualmente com o auxílio de uma rede entomológica, sacrificadas em um frasco contendo éter e transferidas para frascos numerados. Local e data da coleta, horário de captura e essência visitada foram registrados.

Foram capturados no total 2.511 indivíduos, sendo apenas seis de Eufriesea laniventris. Cinco indivíduos foram coletados em fragmentos urbanos: quatro no Parque do Mindu, em salicilato de metila, no dia 8 de agosto e um no INPA - CPAQ, em 1,8 cineol, no dia 28 de agosto. Apenas um foi coletado em mata primária, na Estação Experimental de Silvicultura Tropical, em salicilato de metila, no dia 6 de agosto. Todos os indivíduos foram coletados entre 10:00 e 11:00 horas.

Todos os exemplares estão depositados na coleção entomológica do INPA, Manaus, Amazonas.

A distribuição geográfica de $E$. laniventris ainda não é bem conhecida. A localidade do tipo é Belém, Pará, Brasil (Moure, 1967: Kimsey, 1982; Kimsey \& Dressler, 1986) e, em sua revisão do gênero, Kimsey (1982) cita sua ocorrência apenas na Venezuela: Bolívar (Santa Maria de Erebato); Suriname: Albina e no Brasil: Pará. Por outro lado, sua ocorrência não tem sido registrada na Amazônia peruana (Dressler, 1984) e na Colômbia (Bonilla-Gómes \& Nates-Parra, 1992; Ramírez et al., 2002), muito embora os últimos autores acrescentem sua ocorrência na Guiana e em Trinidad.

Nenhum trabalho realizado anteriormente na região de Manaus havia detectado a presença dessa espécie (Braga, 1976; Powell \& Powell, 1987; Becker et al., 1991; Morato et al., 1992; Morato (1994), Oliveira \& Campos, 1995 e Oliveira 2001), apesar do grande número de espécimes coletados.

Como as espécies desse gênero são reconhecidamente sazonais (Silveira et al., 2002), esse pode ser o motivo desta não ter sido detectada até então.

\section{AGRADECIMENTOS}

Ao Dr. Roger William Hutchings Honegger (CPEC - INPA) pela correção do abstract. Ao MCT/INPA através dos Programas de Pesquisas Institucionais (PPIs 1-3030 e 1-3590) pelo auxílio financeiro.

\section{BIBLIOGRAFIA CITADA}

Becker, P.; Moure, J.S.; Peralta, F.J.A. 1991. More about euglossine bees in amazonian forest fragments. Biotropica, 23(4b): 586-591.

Bonilla-Gómez, M.A.; Nates-Parra, G. 1992. Abejas euglosinas de Colombia (Hymenoptera: Apidae). I. Claves ilustradas. Caldasia, 17 (01):149-172.
Braga, P.I.S. 1976. Atração de abelhas polinizadoras de Orchidaceae com auxílio de iscas-odores na campina, campinarana e floresta tropical unida na região de Manaus. Ciência e Cultura, 28(7): 767-773.

Dressler, R.L. 1984. Euglossine bees (Hymenoptera: Apidae) of the Tambopata Reserved Zone, Madre de Dios, Perú. Revista Peruana de Entomología, 27: 75-79.

Kimsey, L.S. 1982. Systematics of bees of the genus Eufriesea (Hymenoptera, Apidae). University of California Press, Berkeley, CA, USA. 125pp.

Kimsey, L.S.; Dressler, R.L. 1986. Synonymic species list of Euglossini. Pan-Pacific Entomologist, 62(3): 229-236.

Morato, E.F. 1994. Abundância e riqueza de machos de Euglossini (Hymenoptera: Apidae) em mata de terra firme e áreas de derrubada, nas vizinhanças de Manaus (Brasil). Boletim do Museu Paraense Emílio Goeldi, série Zoologia, 10(1): 95-105.

Morato, E.F.; Campos, L.A.O.; Moure, J.S. 1992. Abelhas Euglossini (Hymenoptera: Apidae) coletadas na Amazônia central. Revista Brasileira Entomologia, 36(4): 767-771.

Moure, J.S. 1967. A check-list of the known euglossine bees (Hymenoptera, Apidae). Atas do Simpósio da Biota Amazônica, Zoologia 5: 395-415.

Oliveira, M. L. 2001. Stingless bees (Meliponini) and orchid bees (Euglossini) in terra firme tropical forests and forests fragments. In: Bierregaard Jr., R.O.; Gascon, C.; Lovejoy, T.E.; Mesquita, R. (Eds.). Lessons from Amazonia: The ecology and conservation of a fragmented forest. Yale University Press, New Haven, USA. p. 208-218.

Oliveira, M.L.; Campos, L.A.O. 1995. Abundância, riqueza e diversidade de abelhas Euglossinae (Hymenoptera, Apidae) em florestas contínuas de terra firme na Amazônia Central, Brasil. Revista brasileira Zoologia, 12(3): 547-556.

Powell, A.; Powell, N.V. 1987. Population dynamics of male euglossine bees in amazonian forest fragments. Biotropica, 19(2)176-179.

Ramírez, S., Dressler, R.L. \& Ospina, M. 2002. Abejas euglosinas (Hymenoptera: Apidae) de la región Neotropical: listado de especies com notas sobre su biología. Biota Colombiana, 3(1): 7-118.

Silveira, F.A.; Melo, G.A.R.; Almeida, E.A.B. 2002. Abelhas brasileiras. Sistemática e identificação. Fundação Araucária, Belo Horizonte. 253pp.

\section{RECEBIDO EM 09/09/2003 ACEITO EM 28/11/2003}

